\title{
APPLICATION OF CERTAIN FRACTIONAL CALCULUS OPERATORS IN STATISTICAL DISTRIBUTIONS
}

\author{
R. K. RAINA AND MAMTA BOLIA
}

\begin{abstract}
This paper deals with the application of certain classes of fractional calculus operators in statistical distributions. The images of product combinations of special functions under the calculus of operators are applied to certain generalized forms of univariate and multivariate statistical distributions. Further results giving the expectations, cummulative functions and characteristic functions of such special function distributions are also obtained.
\end{abstract}

\section{Introduction and Prelimanaries}

The theory of fractional calculus is receiving increasing attention from many researchers. After the celebrated conference at the university of New Haven (USA) in 1974 [9], several papers on different aspects relating fractional calculus and their applications have appeared including the two international conferences at the University of Strathclyde (U.K.) in 1984 and at Nihon University (Japan) in 1989. Three recent books on the subject by Miller and Ross.[8], Kiryakova [6] and Samko, Kilbas and Marichev [13] give fairly good account of the development in fractional calculus and their applications to various problems of analysis. Applications of fractional calculus operators in problems of statistics have been considered in [9], [12] and [13].

The present paper is intended to apply fractional calculus operators to certain univariate and multivariate statistical distributions. We first state few formulas giving the images under fractional calculus operators of the product combinations of elementary functions and hypergeometrie functions which are expressed in terms of generalised Kampe' de Féreit functions [15]. These results are then applied to certain classes of statistical distributions. Further results concerning the problems of finding expectations.

Received May 10, 1994; revised Octember 15, 1994.

1991 Mathematics Subject Classification. Primary 26A 33, 33C20, secondary 62E10.

Key words and phrases. Fractional calculus operators, univariate and multivariate distributions, Charecteristic functions, Cummulative functions. 
Cummulative functions and characterstic functions of our special function distributions are also obtained.

Let $\alpha, \beta, \eta \in C$ with $\operatorname{Re}(\alpha)>0$. The fractional integral of the first kind of a suitable function $f(x)$ is defined by (see[10]):

$$
I_{o, x}^{\alpha, \beta, \eta} f(x)=\frac{x^{-\alpha-\beta}}{\Gamma(\alpha)} \int_{o}^{x}(x-t)^{\alpha-1} F\left(\alpha+\beta,-\eta ; \alpha ; 1-\frac{t}{x}\right) f(t) d t
$$

for $x \in R_{+}$.

Here and elsewhere, $C$ is the complex number field, $R_{+}=(0, \infty), N_{0}$ denotes the set of nonnegative integers. The fractional derivative of $f(x)$ for $\operatorname{Re}(\alpha)<0$ is defined by

$$
I_{o, x}^{\alpha, \beta, \eta} f(x)=\frac{d^{n}}{d x^{n}} I_{o, x}^{\alpha+n, \beta-n, \eta-n} f(x)
$$

provided that $o<\operatorname{Re}(\alpha)+n \leq 1, n \in N_{0}$.

The Gauss hypergeometric function $F(a, b ; c ; z)$ appearing in (1.1) is a special case of the generalised hypergeomertric function

$$
{ }_{p} F_{q}\left(a_{1}, \ldots, a_{p} ; b_{1}, \ldots, b_{q} ; z\right)=\sum_{m=0}^{\infty} \frac{\prod_{j=1}^{p}\left(a_{j}\right)_{m}}{\prod_{j=1}^{q}\left(b_{j}\right)_{m}} \cdot \frac{z^{m}}{m !}
$$

where $(a)_{n}=\frac{\Gamma(a+n)}{\Gamma(a)}\left(n \in N_{0}\right)$, denotes the usual Pochhammer symbol and ${ }_{2} F_{1}(a, b ; c ; z)$ $=F(a, b ; c ; z)$. In our sequel we shall use the generalized Kampé de Fériet function $[15, \mathrm{p} .38]$ defined by

$$
\begin{aligned}
& F_{q: q_{1}, \ldots, q_{n}}^{p: p_{1}, \ldots, p_{n}}\left[\begin{array}{l}
\left.\left(a_{p}\right):\left(b_{p_{1}}^{1}\right) ; \ldots ;\left(b_{p_{n}}^{n}\right) ; x_{1}, \ldots, x_{n}\right] \\
\left(\alpha_{q}\right) ;\left(\beta_{q_{1}}^{1}\right) ; \ldots ;\left(\beta_{q_{n}}^{n}\right) ;
\end{array} x_{1}\right] \\
& =\sum_{s_{1}, \ldots, s_{n}=0}^{\infty} \phi\left(s_{1}, \ldots, s_{n}\right) \frac{x_{1}^{s_{1}}}{s_{1} !} \ldots \frac{x_{n}^{s_{n}}}{s_{n} !},
\end{aligned}
$$

where

$$
\phi\left(s_{1}, \cdots, s_{n}\right)=\frac{\prod_{j=1}^{p}\left(a_{j}\right)_{s_{1}+\cdots+s_{n}} \prod_{j=1}^{p_{1}}\left(b_{j}^{1}\right)_{s_{1}} \cdots \prod_{j=1}^{p_{n}}\left(b_{j}^{n}\right)_{s_{n}}}{\prod_{j=1}^{q}\left(\alpha_{j}\right)_{s_{1}+\cdots+s_{n}} \prod_{j=1}^{q_{1}}\left(\beta_{j}^{1}\right)_{s_{1}} \cdots \prod_{j=1}^{q_{n}}\left(\beta_{j}^{n}\right)_{s_{n}}}
$$

for $p+p_{k} \leq q+q_{k}+1(k=1, \ldots, n)$. The symbol $\left(a_{p}\right)$ means the p-dimensional vector of complex parameters $\left(a_{1}, \ldots, a_{p}\right)$ with similar interpretations for $\left(\alpha_{q}\right),\left(a_{p_{1}}^{1}\right)$ etc., and it being assumed that none of the denominator parameters in (1.4) is equal to zero or negative integer. 


\section{Images Under Fractional Calculus Operators.}

Throughout this paper we will denote $k \in A(\beta, \eta)$ if $k, \beta, \eta \in C$ satisfy the inequality

$$
\operatorname{Re}(k)>\max [o, \operatorname{Re}(\beta-\eta)]-1 \text {. }
$$

We state below the formulas related to fractional calculus operators (1.1) and (1.2) which are needed in our sequel:

\section{Lemma 1.}

If $\lambda-1 \in A(\beta, \eta), p_{i} \leq q_{i}$ and $\left(p_{i}=q_{i}+1, \max \left|r_{i} x\right|<1\right), \forall i \in\{1, \ldots, n\}$, then

$$
\begin{aligned}
& I_{o, x}^{\alpha, \beta, \eta}\left(x^{\lambda-1} \prod_{i=1}^{n}\left\{{ }_{p_{i}} F_{q_{i}}\left[\begin{array}{c}
\left(a_{p_{i}}^{i}\right) ; \\
\left(b_{q_{i}}^{i}\right) ;
\end{array} r_{i} x\right]\right\}\right) \\
= & \frac{\Gamma(\lambda) \Gamma(\lambda-\beta+\eta)}{\Gamma(\lambda-\beta) \Gamma(\lambda+\alpha+\eta)} x^{\lambda-\beta-1} \\
& . F_{2: q_{1} ; \ldots ; q_{n}}^{2: p_{1} ; \ldots p_{n}}\left[\begin{array}{ll}
\lambda, \lambda-\beta+\eta & :\left(a_{p_{1}}^{1}\right) ; \ldots ;\left(a_{p_{n}}^{n}\right) ; r_{1} x, \ldots, r_{n} x \\
\lambda-\beta, \lambda+\alpha+\eta & :\left(b_{q_{1}}^{1}\right) ; \ldots ;\left(b_{q_{n}}^{n}\right) ;
\end{array}\right.
\end{aligned}
$$

Proof. Expanding each hypergeometric function and then applying the formula $[12$, p.55, Eqn. (2.4)]:

$$
I^{\alpha, \beta, \eta} x^{k}=\frac{\Gamma(1+k) \Gamma(1+k-\beta+\eta)}{\Gamma(1+k-\beta) \Gamma(1+k+\alpha+\eta)} x^{k-\beta}
$$

and interpreting the n-series by means of the definition (1.4), we arrive at the desired result (2.1). Similarly, we can establish the following results:

Lemma 2. If $\lambda-1 \in A(\beta, \eta), \operatorname{Re}(h)>0, \mu \in C$, and $p_{i} \leq q_{i}\left(p_{i}=q_{i}+1, \operatorname{Max}\right.$ $\left.\left|r_{i} x\right|<1\right), \forall i \in\{1, \ldots, n\}$, then

$$
\begin{aligned}
& I_{0, x}^{\alpha, \beta, \eta}\left(x^{\lambda-1}(x+h)^{-\mu} \prod_{i=1}^{n}\left\{{ }_{p_{i}} F_{q_{i}}\left[\begin{array}{l}
\left(a_{p_{i}}^{i}\right) ; \\
\left(b_{q_{i}}^{i}\right) ;
\end{array} r_{i} x\right]\right\}\right) \\
= & \frac{\Gamma(\lambda) \Gamma(\lambda-\beta+\eta)}{\Gamma(\lambda-\beta) \Gamma(\lambda+\alpha+\beta)} x^{\lambda-\beta-1} h^{-\mu} \\
& . F_{2: q_{1} ; \ldots ; q_{n} ; 0}^{2: p_{1} ; \ldots ; p_{n} ; 1}\left[\begin{array}{ll}
\lambda, \lambda-\beta+\eta & :\left(a_{p_{1}}^{1}\right) ; \ldots ;\left(a_{p_{n}}^{n}\right) ;-\mu ; \\
\lambda-\beta, \lambda+\alpha+\eta & :\left(b_{q_{1}}^{1}\right) ; \ldots ;\left(b_{q_{n}}^{n}\right) ;--;
\end{array} r_{1} x, \ldots, r_{n} x,-\frac{x}{h}\right]
\end{aligned}
$$

Lemma 3. If $\lambda-1 \in A(\beta, \eta), \operatorname{Re}(\mu)>0$ and $p_{i} \leq q_{i}\left(p_{i}=q_{i}+1, \max \left|r_{i} x\right|<1\right)$, $\forall i \in\{1, \ldots, n\}$, then

$$
\begin{gathered}
I_{0, x}^{\alpha, \beta, \eta}\left(x^{\lambda-1} e^{\mu x} \prod_{i=1}^{n}\left\{{ }_{p_{i}} F_{q_{i}}\left[\begin{array}{c}
\left(a_{p_{i}}^{i}\right) ; \\
\left(b_{q_{i}}^{i}\right) ;
\end{array} r_{i} x\right]\right\}\right)=\frac{\Gamma(\lambda) \Gamma(\lambda-\beta+\eta)}{\Gamma(\lambda-\beta) \Gamma(\lambda+\alpha+\eta)} x^{\lambda-\beta-1} \\
. F_{2: q_{1}, \ldots, q_{n}}^{2: p_{1}, \ldots, p_{n}}\left[\begin{array}{ll}
\lambda, \lambda-\beta+\eta & :\left(a_{p_{1}}^{1}\right) ; \ldots ;\left(a_{p_{n}}^{n}\right) ; r_{1} x, \ldots, r_{n} x, \mu x \\
\lambda-\beta, \lambda+\alpha+\eta & :\left(b_{q_{1}}^{1}\right) ; \ldots ;\left(b_{q_{n}}^{n}\right) ;
\end{array}\right.
\end{gathered}
$$


Lemma 4. If $\lambda-1 \in A(\beta, \eta), p_{i} \leq q_{i}, A_{j} \leq B_{j}$ and $\left(p_{i}=q_{i}+1, A_{j}=B_{j}+1\right.$, $\left.\max \left|r_{i} x\right|<1, \max \left|s_{j} x\right|<1\right), \forall_{i} \in\{1, \ldots, n\}, j \in\{1, \ldots, m\}$, then

$$
\begin{aligned}
& I_{o, x}^{\alpha, \beta, \eta}\left(x^{\lambda-1} \prod_{i=1}^{n}\left\{{ }_{p_{i}} F_{q_{i}}\left[\begin{array}{l}
\left(a_{p_{i}}^{i}\right) ; \\
\left(b_{q_{i}}^{i}\right) ;
\end{array} r_{i} x\right]\right\} \cdot \prod_{j=1}^{m}\left\{{ }_{A_{j}} F_{B_{j}}\left[\begin{array}{l}
\left(c_{A_{j}}^{j}\right) ; \\
\left(d_{B_{i}}^{j}\right) ;
\end{array} s_{j} x\right]\right\}\right) \\
& =\frac{\Gamma(\lambda) \Gamma(\lambda-\beta+\eta)}{\Gamma(\lambda-\beta) \Gamma(\lambda+\alpha+\eta)} x^{\lambda-\beta-1} F_{2: q_{1} ; \ldots ; q_{n} ; B_{1} ; \ldots ; B_{m}}^{2: p_{1} ; \ldots ; p_{n} ; A_{1} ; \ldots ; A_{m}} \\
& \cdot\left[\begin{array}{ll}
\lambda, \lambda-\beta+\eta & :\left(a_{p^{1}}^{1}\right) ; \ldots ;\left(a_{p_{n}}^{n}\right) ;\left(c_{A_{1}}^{1}\right) ; \ldots ;\left(c_{A_{m}}^{m}\right) ; \\
\lambda-\beta, \lambda+\alpha+\eta & :\left(b_{q^{1}}^{1}\right) ; \ldots ;\left(b_{q_{n}}^{n}\right) ;\left(d_{B_{1}}^{1}\right) ; \ldots ;\left(d_{B_{m}}^{m}\right) ;
\end{array}\right.
\end{aligned}
$$

Remark 1. The above results (Lemmas 1-4) can also be deduced from the formulas giving the fractional calculus operator images of more involved higher classes of special functions (see[11]).

Remark 2. It may be noted that Lemma 1 can also be obtained from Lemma 2 (or Lemma 3). Lemma 3 would also follow as a limiting case of Lemma 2, and Lemma 4 can be manipulated to yield the formulas (2.1), (2.3) and (2.4).

\section{A Generalized Finite Distribution}

Let us define a family of distributions having the p.d.f. of the form

$$
f(x)= \begin{cases}\Delta^{-1}(x-h)^{p-1}(k-x)^{\alpha-1} F\left(\alpha+\beta,-\eta ; \alpha ; \frac{k-x}{k-h}\right) & \\
\cdot \prod_{i=1}^{n}\left\{{ }_{p_{i}} F_{q_{i}}\left[\begin{array}{l}
\left(a_{p_{i}}^{i}\right) ; \\
\left(b_{q_{i}}^{i}\right) ;
\end{array} r_{i}(k-h)\right]\right\} & \text { for } h \leq x \leq k, \\
0, & \text { otherwise }\end{cases}
$$

$\operatorname{Re}(\alpha)>0, \beta, \eta \in C, p-1 \in A(\beta, \eta)$ and $\max _{1 \leq j \leq n}\left|r_{j}(k-h)\right|<1(h \neq k)$,

where

$$
\begin{aligned}
\Delta= & \frac{\Gamma(\alpha) \Gamma(p) \Gamma(p-\beta+\eta)}{\Gamma(p-\beta) \Gamma(p+\alpha+\eta)}(k-h)^{\alpha+p-1} \\
& . F_{2 ; q_{1} ; \ldots ; q_{n}}^{2 ; p_{1} ; \ldots ; p_{n}}\left[\begin{array}{ll}
p, p-\beta+\eta & :\left(a_{p_{1}}^{1}\right) ; \ldots ;\left(a_{p_{n}}^{n}\right) ; \\
p-\beta, p+\alpha+\eta & :\left(b_{q_{1}}^{1}\right) ; \ldots ;\left(b_{q_{n}}^{n}\right) ;
\end{array} r_{1}(k-h), \ldots, r_{n}(k-h)\right] .
\end{aligned}
$$

We may mention here that the statistical distributions involving the Gaussian hypergeometric function ${ }_{2} F_{1}$ in the p.d.f. were earlier studied by Dyer [1] and Mathai and Saxena [7]. The use of the generalized hypergeometric function ${ }_{p} F_{q}$ in statistical distributions have also been investigated, see [14]. It seems worthwhile and of importance here to describe the motivation and usefulness behind studying the family of distributions 
such as the one considered in (3.1) above. Many classical distributions are known to be associated with the Beta and Gamma distributions. A large number of such distribution functions involving either one or several variates are expressible in terms of hypergeometric functions of one or more variables. To consider a specific example, it is pointed out in [15] that if $U_{j}(j=1,2)$ have a Gamma distribution with space parameter $\phi_{j}$ and scalar parameter $B_{j}$, then the ratio $X=U_{1} / U_{2}\left(U_{1}\right.$ and $U_{2}$ are independent) is a generalized $F$ variate, and the distribution of $X$ is then called the generalized $F$ distribution with the p.d.f. given by

$$
f(x)=\frac{\alpha^{p}}{\beta(p, m-p)} \frac{x^{p-1}}{(1+\alpha x)^{m}}(x>0, \alpha>0, m>p>0)
$$

where

$$
\alpha=\frac{\beta_{2}}{\beta_{1}}, p=\phi_{1}, m=\phi_{1}+\phi_{2} .
$$

Dyer [1] showed that if a random variable $X$ has the p.d.f. given by (3.3), then the distribution function of such a random variable can easily be expressed in terms of the hypergeometric function ${ }_{2} F_{1}$, and the distribution function $Y=X_{1}+X_{2}$ (where $X_{1}$ and $X_{2}$ are independent variates having p.d.f. of the form (3.3)) involves the triple hypergeometric series $F_{T}([15, p .43, E q n .(10)]$. One may refer to [16,p.264] for another instance of the occurrence of multiple hypergeometric series in probability theory.

Further, large cases of statistical problems involve the distributions of linear combinations of random variables, where individual variates are assumed to have particular types of distributions. For instance, if we consider the total service time required in a routine automobile engine check-up, then the total service time required is a linear combination of random variables. In order to make inferential statements about the total service time, one needs the distribution of linear combinations of random variables. These problems are closely related to fields of queueing theory and other areas of operations research, like those encountered in inter-live-birth control (see[5]). Also, in many situations joint distributions are required to be found which involve bivariate (or multivariate) special functions (see [3] and [4]). Hence, the family of distributions considered in (3.1) may thus be looked upon as a general class of finite distributions which may be used to various statistical situations as elucidated above.

To show that (3.1) represents a p.d.f., we find that

$$
\begin{aligned}
& \int_{-\infty}^{\infty} f(x) d x=\int_{h}^{k} f(x) d x \\
= & \Delta^{-1} \int_{h}^{k}(x-h)^{p-1}(k-x)^{\alpha-1} F\left(\alpha+\beta,-\eta ; \alpha ; \frac{k-x}{k-h}\right) \prod_{i=1}^{n}\left\{p _ { i } F _ { q _ { i } } \left[\begin{array}{c}
\left(a_{p_{i}}^{i}\right) ; \\
\left(b_{q_{i}}^{i}\right) ;
\end{array}\right.\right.
\end{aligned}
$$

A simple change of variable puts (3.3) in the operator form as

$$
\int_{h}^{k} f(x) d x=\Delta^{-1} \Gamma(\alpha) u^{\alpha+\beta} I_{o, u}^{\alpha, \beta, \eta}\left(u^{p-1} \prod_{j=1}^{n}\left\{{ }_{p_{i}} F_{q_{i}}\left[\begin{array}{l}
\left(a_{p_{i}}^{i}\right) ; \\
\left(b_{q_{i}}^{i}\right) ;
\end{array} r_{i} u\right]\right\}\right)
$$


Applying Lemma 1, we at once arrive at the value unity on the right side showing that (3.1) is indeed a p.d.f.

\section{Expectation of Function}

For any function $g(x)$, the expectation of $g(x)$ with respect to the p.d.f. $f(x)$ is defined by

$$
E\{g(x)\}=\int_{-\infty}^{\infty} f(x) g(x) d x .
$$

Consider the function $g(x)$ in terms of products of m-generalized hypergeometric functions given by

$$
g(x)=\prod_{j=1}^{m}\left\{A_{j} F_{B_{j}}\left[\begin{array}{l}
\left(c_{A_{j}^{j}}^{j}\right) ; \\
\left(d_{B_{j}}^{j}\right) ;
\end{array} s_{j}(x-h)\right]\right\},
$$

with $\max _{1 \leq j \leq m}\left|s_{j}(k-h)\right|<1$. Let a p.d.f. $f(x)$ be defined by (3.1). We have then

$$
\begin{array}{r}
E\{g(x)\}=\Delta^{-1} \Gamma(\alpha) u^{\alpha+\beta} I_{0, u}^{(\alpha, \beta, \eta)}\left(u^{p-1} \prod_{i=1}^{n}\left\{{ }_{p_{i}} F_{q_{i}}\left[\begin{array}{l}
\left(a_{p_{i}}^{i}\right) ; \\
\left(b_{q_{i}}^{i}\right) ;
\end{array} r_{i} u\right]\right\}\right. \\
\left.\cdot \prod_{j=1}^{m}\left\{A_{j} F_{B_{j}}\left[\begin{array}{l}
\left(c_{A_{j}}^{j}\right) ; \\
\left(d_{B_{j}}^{j}\right) ;
\end{array} s_{j} u\right]\right\}\right)
\end{array}
$$

where $u=k-h$. An application of Lemma 4 yields

$$
\begin{aligned}
& E\{g(x)\}=\nabla^{-1} F_{2: q_{1} ; \ldots ; q_{n} ; B_{1} ; \ldots ; B_{m}}^{2: p_{1} ; \ldots ; p_{n} ; A_{1} ; \ldots ; A_{m}} \\
& \cdot\left[\begin{array}{ll}
p, p-\beta+\eta & \left.:\left(a_{p_{1}}^{1}\right) ; \ldots ;\left(a_{p_{n}}^{n}\right) ;\left(c_{A_{1}}^{1}\right) ; \ldots ;\left(c_{A_{m}}^{m}\right) ; r_{1} u, \ldots, r_{n} u, s_{1} u, \ldots, s_{m} u\right] \\
p-\beta, p+\alpha+\eta & :\left(b_{q_{1}}^{1}\right) ; \ldots ;\left(b_{q_{n}}^{n}\right) ;\left(d_{B_{1}}^{1}\right) ; \ldots ;\left(d_{B_{m}}^{m}\right) ;
\end{array}\right. \\
& \nabla=F_{2: q_{1} ; \ldots ; q_{n}}^{2: p_{1} ; \ldots ; p_{n}}\left[\begin{array}{ll}
p, p-\beta+\eta & :\left(a_{p_{1}}^{1}\right) ; \ldots ;\left(a_{p_{n}}^{n}\right) ; \\
p-\beta, p+\alpha+\eta & :\left(b_{q_{1}}^{1}\right) ; \ldots ;\left(b_{q_{n}}^{n}\right) ;
\end{array} r_{1} u, \ldots, r_{n} u\right]
\end{aligned}
$$

\section{Cummulative Function}

The cummulative probability function for the distrbution function $F(t)$ is given by

$$
F(t)=\int_{-\infty}^{t} f(x) d x
$$

For the p.d.f. $f(x)$ defined by (3.1) and for $h \leq t \leq k$. we have

$$
\begin{aligned}
& F(t)=\int_{-\infty}^{t} f(x) d x \\
= & \Delta^{-1} \int_{h}^{t}(x-h)^{p-1}(k-x)^{\alpha-1} F\left(\alpha+\beta,-\eta ; \alpha ; \frac{k-x}{k-h}\right) \prod_{i=1}^{n}\left\{{ }_{p_{i}} F_{q_{i}}\left[\begin{array}{c}
\left(a_{p_{i}}^{i}\right) ; \\
\left(b_{q_{i}}^{i}\right) ;
\end{array} r_{i}(x-h)\right]\right\} d x .
\end{aligned}
$$


This gives

$$
\begin{array}{r}
F(t)=\Delta^{-1} \int_{0}^{t-h} z^{p-1}(u-z)^{\alpha-1} F\left(\alpha+\beta,-\eta ; \alpha ; \frac{u-z}{u}\right) \\
\cdot \prod_{i=1}^{n}\left\{{ }_{p_{i}} F_{q_{i}}\left[\begin{array}{c}
\left(a_{p_{i}}^{i}\right) ; \\
\left(b_{q_{i}}^{i}\right) ;
\end{array} r_{i} z\right]\right\} d z
\end{array}
$$

where $u=k-h$. To evaluate the integral occurring on the R.H.S. of (5.2), we proceed as follows (see also [12]):

Making use of the continuation formula of the Gauss function

$$
\begin{aligned}
& F(a, b ; c ; z)= \frac{\Gamma(c) \Gamma(c-a-b)}{\Gamma(c-a) \Gamma(c-b)} F(a, b ; a+b-c+1 ; 1-z) \\
&+\frac{\Gamma(c) \Gamma(a+b-c)}{\Gamma(a) \Gamma(b)}(1-z)^{c-a-b} F(c-a, c-b ; c-a-b+1 ; 1-z) \\
&|\arg (1-z)|<\pi
\end{aligned}
$$

and the Eulerian transformation

$$
F(a, b ; c ; z)=(1-z)^{c-a-b} F(c-a, c-b ; c ; z), \quad|\arg (1-z)|<\pi,
$$

in the two terms of the resulting R.H.S. of (5.2). expanding the Gauss functions and then integrating term by term. we get

$$
\begin{aligned}
F(t)= & \Delta^{-1} \frac{\Gamma(p-\beta) \Gamma(\eta-\beta) \Gamma(p+\alpha+\eta)}{\Gamma(-\beta) \Gamma(\alpha+\eta) \Gamma(p+\eta-\beta) \Gamma(p+1)}\left(\frac{t-h}{u}\right)^{p} F_{1: q_{1} ; \ldots ; q_{n} ; 1}^{1: p_{1} ; \ldots ; p_{n} ; 2} \\
& \cdot\left[\begin{array}{lll}
p \quad:\left(a_{p_{1}}^{1}\right) ; \ldots ;\left(a_{p_{n}}^{n}\right) ; \quad-\eta-\alpha+1, \beta+1 ; & \left.r_{1}(t-h), \ldots, r_{n}(t-h), \frac{t-h}{u}\right] \\
p+1 \quad:\left(b_{q_{1}}^{1}\right) ; \ldots ;\left(b_{q_{n}}^{n}\right) ; \quad \beta-\eta+1 ;
\end{array}\right. \\
& +\nabla^{-1} \frac{\Gamma(\beta-\eta) \Gamma(p-\beta) \Gamma(p+\alpha+\eta)}{\Gamma(-\eta) \Gamma(\alpha+\beta) \Gamma(p-\beta+\eta+1) \Gamma(p)}\left(\frac{t-h}{u}\right)^{p-\beta+\eta} \\
& F_{1: q_{1} ; \ldots ; q_{n} ; 1}^{1: p_{1} ; \ldots ; p_{n} ; 2}\left[\begin{array}{ll}
p-\beta+\eta & :\left(a_{p_{1}}^{1}\right) ; \ldots ;\left(a_{p_{n}}^{n}\right) ; \quad \eta+1,-\beta-\alpha+1 ; \\
p-\beta+\eta+1 \quad:\left(b_{q_{1}}^{1}\right) ; \ldots ;\left(b_{q_{n}}^{n}\right) ; \quad-\beta+\eta+1 ;
\end{array}\right],
\end{aligned}
$$

where $u=k-h$, and $\nabla$ is given by (4.5).

\section{Charecteristic Function}

The Charecteristic function $\phi(t)$ of a random variable $x$ with respect to a p.d.f. $f(x)$ is defind by

$$
\phi(t)=E\left\{e^{i t x}\right\}=\int_{-\infty}^{\infty} e^{-i t x} f(x) d x .
$$


For the p.d.f. $f(x)$ defined by (3.1), we have

$$
\begin{aligned}
& \phi(t)=\Delta^{-1} \int_{h}^{k} e^{i t x}(x-h)^{p-1}(k-x)^{\alpha-1} F\left(\alpha+\beta,-\eta ; \alpha ; \frac{k-x}{k-h}\right) \\
& \prod_{i=1}^{n}\left\{{ }_{p_{i}} F_{q_{i}}\left[\begin{array}{l}
\left(a_{p_{i}}^{i}\right) ; \\
\left(b_{q_{i}}^{i}\right) ;
\end{array} r_{i}(x-h)\right]\right\} d x .
\end{aligned}
$$

A change of variable transforms (6.2) in the following operator from:

$$
\phi(t)=\Delta^{-1} \Gamma(\alpha) u^{\alpha+\beta} e^{i h t} I_{o, u}^{\alpha, \beta, \eta}\left(u^{p-1} e^{i t u} \prod_{i=1}^{n}\left\{p_{i} F_{q_{i}}\left[\begin{array}{l}
\left(a_{p_{p}}^{i}\right) ; \\
\left(b_{q_{i}}^{i}\right) ;
\end{array} r_{i} u\right]\right\}\right)
$$

where $u=k-h(k \neq h)$. By appealing to Lemma 3, we get the characteristic function

$$
\begin{aligned}
& \phi(t)=e^{i h t} F_{2: q_{i} ; \ldots ; q_{n}}^{2: p_{i} ; \ldots ; p_{n}} \\
& \cdot\left[\begin{array}{ll}
p, p-\beta+\eta & \left.:\left(a_{p_{1}}^{1}\right) ; \ldots ;\left(a_{p_{n}}^{n}\right) ; \cdot r_{1}(k-h), \ldots, r_{n}(k-h), i t(k-h)\right] \\
p-\beta, p+\alpha+\eta & :\left(b_{q_{1}}^{1}\right) ; \ldots ;\left(b_{q_{n}}^{n}\right) ;
\end{array}\right. \\
& \cdot F_{2: q_{1} ; \ldots ; q_{n}}^{2: p_{1} ; \ldots ; p_{n}}\left[\begin{array}{ll}
p, p-\beta+\eta & :\left(a_{p_{1}}^{1}\right) ; \ldots ;\left(a_{p_{n}}^{n}\right) ; \\
P-\beta, p+\alpha+\eta & :\left(b_{q_{1}}^{1}\right) ; \ldots ;\left(b_{q_{n}}^{n}\right) ;
\end{array} r_{1}(k-h), \ldots, r_{n}(k-h)\right]^{-1}
\end{aligned}
$$

\section{Multivariate Distribution}

In this concluding section we present an extended form of the Dirichlet distribution [3,p.222]. We know that the Liouville's extension of the Dirichlet's result (see[2]) is

$$
\begin{gathered}
\int_{\Omega} \ldots \int x_{1}^{p_{1}-1} \cdots x_{n}^{p_{n}-1} \phi\left(x_{1}+\ldots+x_{n}\right) d x_{1} \ldots d x_{n} \\
\quad=B\left(p_{1}, \ldots, p_{n}\right) \int_{\alpha}^{\beta} z^{\Sigma_{p_{i}}-1} \phi(z) d z
\end{gathered}
$$

for any integrable function $\phi(z)$, where the integrated region $\Omega$ is $\Omega: \alpha \leq x_{1}+\ldots+x_{n} \leq$ $\beta\left(x_{i} \geq 0 ; i=1, \ldots, n\right), 0<\alpha<\beta$. The symbol $\sum$ denotes the summation with respect to $i$ through 1 to $n$, and $B$ means the generalized beta function:

$$
B\left(p_{1}, \ldots, p_{n}\right)=\frac{\Gamma\left(p_{1}\right) \ldots \Gamma\left(p_{n}\right)}{\Gamma\left(p_{1}+\ldots+p_{n}\right)} .
$$

Consider the multivariate density function

$$
f\left(x_{1}, \ldots, x_{n}\right)=\left\{\begin{array}{c}
\omega x_{1}^{p_{1}-1} \ldots x_{n}^{p_{n}-1}\left(\sum x_{i}-\alpha\right)^{p-1}\left(\beta-\sum x_{i}\right)^{\alpha-1} \\
F\left(\lambda+\mu,-\varrho ; \lambda ; \frac{\beta-\sum x_{i}}{\beta-\alpha}\right) \prod_{i=1}^{n}\left\{p_{i} F_{q_{i}}\left[\begin{array}{c}
\left(a_{p_{i}}^{i}\right) ; \\
\left(b_{q_{i}}^{i}\right) ;
\end{array} \quad \sum x_{i}-\alpha\right]\right\} \\
\text { for } \quad \alpha \leq \sum x_{i} \leq \beta \\
\quad \text { elsewhere, }
\end{array}\right.
$$


where $\operatorname{Re}\left(p_{i}\right)>0(i=1, \ldots, n), \operatorname{Re}(p)>0, \lambda, \mu, p \in C, p-1 \in A(\mu, p)$, and

$$
\begin{aligned}
& \omega^{-1}=B\left(p_{1}, \ldots, p_{n}\right) \frac{\Gamma(\lambda) \Gamma(p) \Gamma(p-\mu+p)}{\Gamma(p-\mu) \Gamma(p+\lambda+p)} u^{p+\lambda-1} F_{2: q_{1} ; \ldots ; q_{n}}^{2: p_{1} ; \ldots ; p_{n}} \\
& {\left[\begin{array}{lll}
p, p-\mu+p & :\left(a_{p_{1}}^{1}\right) ; \ldots ;\left(a_{p_{n}}^{n}\right) ; & 1-\sum p_{i} ; u, \ldots, u,-\frac{u}{\alpha} \\
p-\mu, p+\alpha+p & :\left(b_{q_{1}}^{1}\right) ; \ldots ;\left(b_{q_{n}}^{n}\right) ; & -----;
\end{array}\right]}
\end{aligned}
$$

where $u=\beta-\alpha$. To verify that (7.3) represents a p.d.f.,we observe that

$$
\begin{aligned}
& \int_{\Omega} \int f\left(x_{1}, \ldots, x_{n}\right) d x_{1} \ldots d x_{n} \\
= & \omega \int \ldots \int_{x_{1} \geq, \alpha \leq \sum x_{i} \leq \beta} x_{1}^{p_{1}-1} \ldots x_{n}^{p_{n}-1}\left(\sum x_{i}-\alpha\right)^{p-1}\left(\beta-\sum x_{i}\right)^{\lambda-1} \\
& \cdot F\left(\lambda+\mu,-\rho ; \lambda ; \frac{\beta-\sum x_{i}}{\beta-\alpha}\right) \prod_{i=1}^{n}\left\{p _ { i } F _ { q _ { i } } \left[\begin{array}{c}
\left(a_{p_{i}}^{i}\right) ; \\
\left.\left.\left(b_{q_{i}}^{i}\right) ; \sum x_{i}-\alpha\right]\right\} d x_{1} \ldots d x_{n}
\end{array}\right.\right. \\
= & \omega B\left(p_{1} \ldots, p_{n}\right) \int_{\alpha}^{\beta} z^{\Sigma p_{i}-1}(z-\alpha)^{p-1}(\beta-z)^{\lambda-1} F\left(\lambda+\mu,-\rho: \lambda ; \frac{\beta-z}{\beta-\alpha}\right) \\
& \left.\cdot \prod_{i=1}^{n}\left\{p_{i} F_{q_{i}}\left[\begin{array}{l}
\left(a_{p_{i}}^{i}\right) ; \\
\left(b_{q_{i}}^{i}\right) ;
\end{array}\right) \alpha-\alpha\right]\right\} d z \\
= & \omega B\left(p_{1}, \ldots, p_{n}\right) \Gamma(\lambda) u^{\lambda+\mu} I_{o, u}^{\lambda, \mu, \rho}\left(u^{p-1}(u+\alpha)^{\sum p_{i}-1} \cdot \prod_{i=1}^{n}\left\{{ }_{p_{i}} F_{q_{i}}\left[\begin{array}{c}
\left(a_{p_{i}}^{i}\right) ; \\
\left(b_{q_{i}}^{i}\right) ;
\end{array}\right]\right\}\right)
\end{aligned}
$$

where $u=\beta-\alpha$. In view of Lemma 2, we find that the value of R.H.S. of (7.5) is unity by virtue of (7.4). This shows that (7.3) is a p.d.f.

Several other properties of statistical nature can be considered for the multivariate distribution characterised by (7.3), as indicated in [4]. In the concluding remark, it may be mentioned that various useful results in statistical distributions and related problems investigated in [3], [4] and [12] can be deduced from the results presented in this paper.

\section{Acknowledgement}

The authors express their sincerest thanks to the referee for valuable suggestions. The work of the first author was supported, in part, by National Board for Higher Mathematics (DAE), Govt. of India under Grant No.26/6/93-G.

\section{References}

[1] D. Dyer, "The convolution of generalized F distributions," J. Amer. Statis. Assoc. 77(1982), 184189.

[2] A. J. Edwards, A Treatise on Integral Calculus, Chelsea Publ., New york, 1954.

[3] H. Exton, Multiple Hypergeometric Functions and Applications, Ellis Horwood Ltd.. Chichester; Halsted press (John Wiley and sons), New York-London-Sydney-Toronto, 1976. 
[4] H. Exton, Hand Book of Hypergeometric Integrals, Ellis Horwood Ltd., Chichester; Halsted press (John Wiley and Sons), Chichester-New York-Brisbane-Torunto, 1978.

[5] A. George and A. M. Mathai, "A generalised distribution for the inter-live birth interval," Sankhya Ser B 37(1975).332-340.

[6] V. S. Kiryakova, "Generalized Fractional Calculus and Applications," Pitman Research Notes in Math. Series, No.301, Longman Group Ltd. U. K., 1994.

[7] A. M. Mathai and R. K. Saxena, "On generalized hypergeometric distribtuion," Metrika 11(1966), 127-132.

[8] K. S. Miller and B. Ross, An introduction to the Fractional Calculus and Fractional Differential Equations, John Wiley and Sons, New York, 1993.

[9] B. Ross, "A brief history and exposition of the fundamental theory of fractional calculus," in Fractional Calculus and its applications (B. Ross, Ed.), pp.1-36, Springer-Verlag, Berlin / Heidelberg / New York, 1975.

[10] M. Saigo, "A certain boundary value problem for the Euler-Darboux equation," Math. Japon 24(1979), 377-385.

[11] M. Saigo and R. K. Raina, "Fractional Calaulus operators associated with the H-function of several variables," To appear in G.F.B. Riemann: A Mathematical Legacy, Edited by Th. M. Rassias and H. M. Srivastava.

[12] M. Saigo and R. K. Raina, "On the fractional calculus operators involving Gauss's series and its applications to certain statistical distributions." Rev. Tec. Ing. Univ., Zulia, 4(1)(1991), 53-62.

[13] S. G. Samko, A. A. Kilbas and O. I. Marichev, Integrals and Derivatives of Fractional Order and Some of Their Applications, Gordon and Breach, New York, 1993.

[14] R. K. Saxena and P. L. Sethi, "Certain properties of bivariate distributions associated with generalized hypergeometric functions," Canad. J. Statis. 1(1973), 171-180.

[15] H. M. Srivastava and P. W. Karlsson, Multiple Gaussian Hypergeometric Series, Ellis Horwood Ltd., Chichester; Halsted press(John Wiley and Sons Inc.), New York-Chichester-Brisban-Toronto, 1985.

[16] H. M. Srivastava and B. R. K. Kashypap, Special Functions in Queueing Theory and Related Stochastic Processes, Academic Press, New York and London, 1982.

Department of Mathematics, C.T.A.E., Campus Udaipur, Udaiput 313001 (Rajsthan), INDIA.

Department of Mathematics, College of Science,Udaipur, Udaiput 313001 (Rajsthan), INDIA. 